OPEN

SUBJECT AREAS:

OPTICAL PHYSICS

PHOTONIC DEVICES

Received

27 March 2014

Accepted

16 June 2014

Published

3 July 2014

Correspondence and requests for materials should be addressed to

H.L.L. (hailuluo@hnu.

edu.cn) or S.C.W.

(scwen@hnu.edu.cn)

\section{Realization of Tunable Photonic Spin Hall Effect by Tailoring the Pancharatnam-Berry Phase}

\author{
Xiaohui Ling ${ }^{1,3}$, Xinxing Zhou ${ }^{2}$, Weixing Shu ${ }^{2}$, Hailu Luo ${ }^{1,2}$ \& Shuangchun Wen ${ }^{1,2}$
}

${ }^{1}$ Key Laboratory of Optoelectronic Devices and Systems of Ministry of Education and Guangdong Province, College of Optoelectronic Engineering, Shenzhen University, Shenzhen 51 8060, ${ }^{2}$ Key Laboratory for Micro-/Nano-optoelectronic Devices of Ministry of Education, College of Physics and Microelectronic Science, Hunan University, Changsha 410082, China, ${ }^{3}$ Department of Physics and Electronic Information Science, Hengyang Normal University, Hengyang 421002, China.

Recent developments in the field of photonic spin Hall effect (SHE) offer new opportunities for advantageous measurement of the optical parameters (refractive index, thickness, etc.) of nanostructures and enable spin-based photonics applications in the future. However, it remains a challenge to develop a tunable photonic SHE with any desired spin-dependent splitting for generation and manipulation of spin-polarized photons. Here, we demonstrate experimentally a scheme to realize the photonic SHE tunably by tailoring the space-variant Pancharatnam-Berry phase (PBP). It is shown that light beams whose polarization with a tunable spatial inhomogeneity can contribute to steering the space-variant PBP which creates a spin-dependent geometric phase gradient, thereby possibly realizing a tunable photonic SHE with any desired spin-dependent splitting. Our scheme provides a convenient method to manipulate the spin photon. The results can be extrapolated to other physical system with similar topological origins.

pin Hall effect (SHE) is a transport phenomenon in which an electric field applied to spin particles results in a spin-dependent shift perpendicular to the electric field direction ${ }^{1-3}$. It plays an important role in the spintronics since it can provide an effective way for generation, manipulation, and detection of spinpolarized electron which define the main challenges in spintronics applications ${ }^{4-6}$. Photonic SHE manifests itself as the spin-dependent splitting of light ${ }^{7-9}$, which can be regarded as a direct optical analogy of the SHE in electronic systems. Recently, photonic SHE has been proved to be an advantageous metrological tool for characterizing the parameters of nanostructures ${ }^{10,11}$. More importantly, it offers new opportunities for manipulating spin photons and developing next generation of spin-controlled photonic devices as counterparts of recently presented spintronics devices ${ }^{4,12}$. Similar to those in the spintronics devices, one major issue in spin-based photonics is to develop a tunable photonic SHE with any desired spin-dependent splitting for generation, manipulation, and detection of spin-polarized photons.

Photonic SHE is generally believed to be a result of topological spin-orbit interaction which describes the coupling between the spin (polarization) and the trajectory (orbit angular momentum) of light beam propagation. The spin-orbit interaction corresponds to two types of geometric phases: the Rytov-Vladimirskii-Berry phase associated with the evolution of the propagation direction of light ${ }^{7-9}$ and the Pancharatnam-Berry phase (PBP) related to the manipulation with the polarization state of light ${ }^{13,14}$. The spin-orbit interaction associated with the Rytov-Vladimirskii-Berry phase is exceedingly small, and the detection of the corresponding photonic SHE needs multiple reflections $s^{14}$ or weak measurements ${ }^{9,15}$. Recently, the strong spin-orbit interaction related to the PBP in metasurface, a two-dimensional electromagnetic nanostructure, enables one to observe a giant photonic $\mathrm{SHE}^{16-18}$. However, the PBP in the metasurface is fixed and unadjustable, once the nanostructure is manufactured. Despite of recent experimental efforts, it remains a challenge to realize a photonic SHE with a high tunability.

In this work, we report an experimental realization of tunable photonic SHE via tailoring the space-variant PBP contributed by a light beam with transversely inhomogeneous polarization. We show that the space-variant polarization is able to invoke the spin-orbit interaction even when propagating through a homogeneous waveplate and produces the space-variant PBP as the inhomogeneous metasurface does, with a high tunability the latter cannot achieve. As the beam with any desired space-variant polarization can be easily generated by many methods (see Ref. 19 for a review and the references therein), involving the contribution of the incident polar- 
ization distribution to the space-variant PBP will introduce a convenient degree of freedom to manipulate the photonic SHE.

\section{Results}

Theoretical analysis. For an inhomogeneous waveplate or metasurface, the space-variant optical axis direction produces a spatial rotation rate and applies a space-variant $\mathrm{PBP}$ to a light beam that passes through it, due to the spin-orbit interaction ${ }^{12,13}$. This inhomogeneous geometric phase forms a spin-dependent geometric phase gradient and generates a spin-dependent shift $(\Delta k)$ in momentum space [Fig. 1(a)]. Indeed, the inhomogeneous birefringent waveplate is not the sole way to produce the PBP. Providing the incident beam takes a spatial rotation rate, namely exhibits an inhomogeneous polarization, the PBP and spin-dependent momentum splitting should still occur [Fig. 1(b)]. Recent works about the spindependent splitting occurring at metasurfaces ${ }^{16-18}$ can be seen as special cases where only the waveplate is spatially inhomogeneous. The scheme proposed here show that a beam with tunable inhomogeneous polarization can also generate the spin-dependent momentum shift even though it passes through a homogenous waveplate.

We now develop a unified theoretical model of the photonic SHE for either the polarization of light beam or the waveplate exhibits a spatial inhomogeneity. First, we consider that only the waveplate is inhomogeneous. It is assumed that the waveplate is a uniaxial crystal with its optical axis direction specified by a space-variant angle $\alpha(r, \varphi)$ $=q \varphi+\alpha_{0}$, where $q$ is a topological charge, $\varphi=\arctan (y / x)$ the local azimuthal angle, and $\alpha_{0}$ the initial angle of local optical axis direction $^{20}$. Some examples of the inhomogeneous waveplate geometries are shown in Fig. 2(a). It is the spatial version of a rotationally homogeneous waveplate ${ }^{13}$. Similar to the temporal rotation rate, the spatial rotation rate of the inhomogeneous waveplate in the polar coordinate is $\Omega_{\alpha}=\mathrm{d} \alpha(r, \varphi) / \mathrm{d} \xi$, where $\mathrm{d} \xi=r \mathrm{~d} \varphi$ is the rotating route with $r$ the radius of the waveplate.
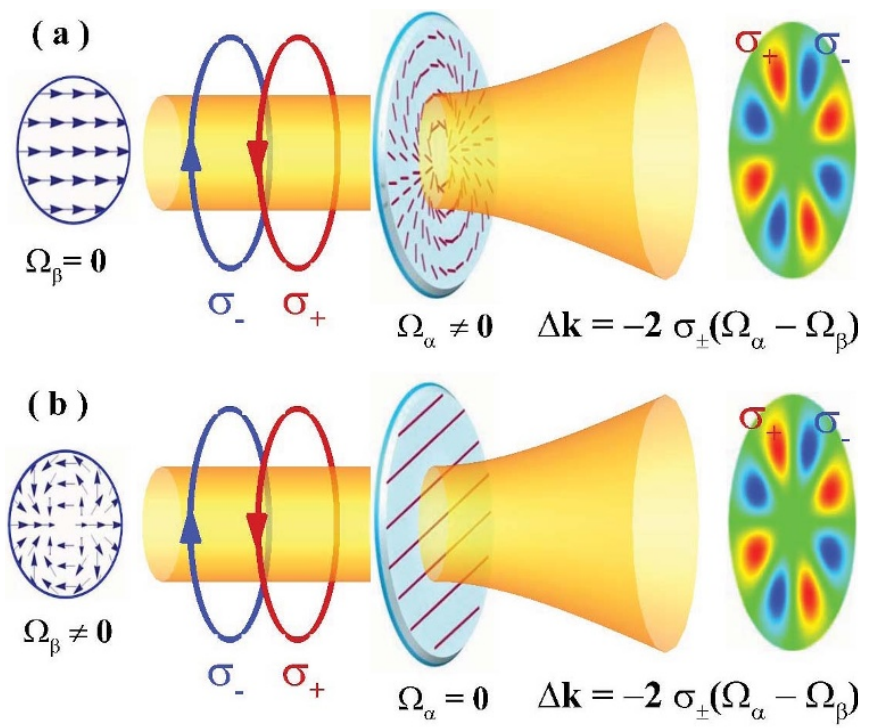

Figure 1 Schematic illustration of the photonic SHE with multi-lobe, spin-dependent momentum splitting. (a) A collimated Gaussian beam with homogeneous polarization (spatial rotation rate $\Omega_{\beta}=0$ ) propagates through an anisotropic metasurface with spatial inhomogeneity $\left(\Omega_{\alpha} \neq 0\right)$. The geometric phase gradient along the azimuthal direction refracts light in twisted directions and results in the spin-dependent momentum splitting. (b) A beam with tunable spatial rotation rate $\left(\Omega_{\beta} \neq 0\right)$ passes through a homogeneous anisotropic waveplate $\left(\Omega_{\alpha}=0\right)$, which can also induce a geometric phase gradient and momentum splitting.

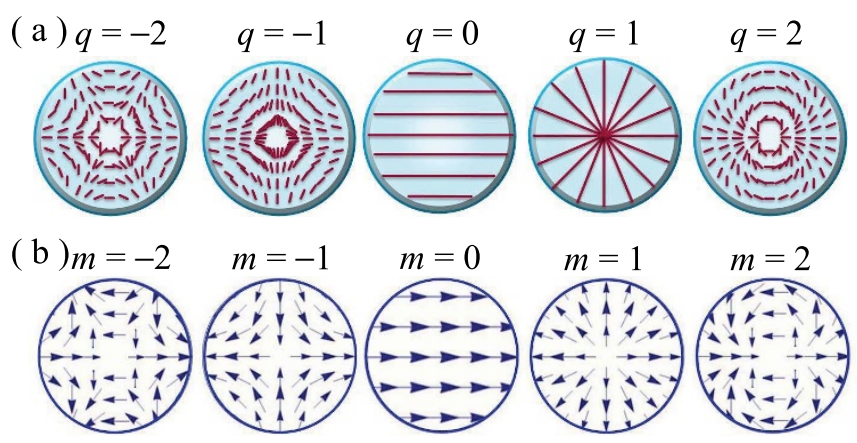

Figure $2 \mid$ (a) Schematic examples of the waveplate geometries for different spatial inhomogeneity. The tangent to the lines shown indicates the direction of local optical axis. (b) Schematic examples of the polarization of CVBs for different spatial inhomogeneity. The arrows represent the local polarization directions.

Next, we consider only the polarization of the incident beam exhibits a spatial inhomogeneity. A linear polarization, with its electric field described by a Jones vector, is given as

$$
E_{\text {in }}(x, y)=\left(\begin{array}{c}
\cos \beta \\
\sin \beta
\end{array}\right) E_{0}(x, y),
$$

where $\beta$ is the polarization angle and $E_{0}(x, y)$ the complex amplitude. Generally, $\beta$ is a constant, and Eq. (1) represents homogeneous linear polarizations with $\beta=0$ and $\pi / 2$ respectively indicating linear $x$ - and $y$-polarizations.

If $\beta$ is a position-dependent function, e.g., $\beta(r, \varphi)=m \varphi+\beta_{0}$, Eq. (1) represents a beam with its polarization having cylindrical symmetry, i.e., the cylindrical vector beam (CVB). Here, $m$ is also a topological charge and $\beta_{0}$ the initial polarization angle for $\varphi=0$. Similar to the inhomogeneous anisotropic waveplate, the inhomogeneous linear polarization has a spatial rotation rate $\Omega_{\beta}=\mathrm{d} \beta(r, \varphi)$ / $\mathrm{d} \xi$ which can be tailored by suitably designing the polarization distribution of the CVBs [see Fig. 2(b) for examples].

In the most general case, both the polarization of light and the waveplate possess spatial rotation rates. Their relative rotation rate can be regarded as $\Omega_{\gamma}=\Omega_{\alpha}-\Omega_{\beta}$. Analogous to the rotational Doppler effect, the PBP can be written as ${ }^{13}$

$$
\Phi_{\mathrm{PB}}=-2 \sigma_{ \pm} \int \Omega_{\gamma} \mathrm{d} \xi=-2 \sigma_{ \pm}\left[(q-m) \varphi+\left(\alpha_{0}-\beta_{0}\right)\right],
$$

where $\sigma_{+}=+1$ and $\sigma_{-}=-1$ representing the left and right circular polarization photons, respectively. Note that the PBP has a spindependent spiral structure which is induced by the topological spin-orbit interaction ${ }^{16,21,22}$. The factor -2 arises because the spin angular momentum vector of photon is reversed, leading to the conversion of spin angular momentum to its orbital parts twice the magnitude of the photons spin angular momentum ${ }^{23,24}$. This indicates that the PBP for beams evolving in a system with nonzero spatial rotation rate would cause a spin-dependent splitting in $k$ (momentum) space, i.e., a manifestation of the photonic SHE.

Unambiguously, the PBP is locally varying, and creates a geometric phase gradient which can be tunable by tailoring the waveplate geometry and polarization distribution of the CVB. This will lead to a spin-dependent shift in $k$ space. Under the normal incidence, the spin-dependent shift $\Delta k=\Delta k_{\mathrm{r}}+\Delta k_{\varphi}$ can be calculated as the gradient of the PBP:

$$
\Delta k=\nabla \Phi_{\mathrm{PB}}=-2 \sigma_{ \pm}(q-m) \hat{e}_{\varphi},
$$

where $\hat{e}_{\mathrm{r}}\left(\hat{e}_{\varphi}\right)$ is the unit vector in the radial (azimuthal) direction. Here $\Delta k$ only has the azimuthal component $\hat{e}_{\varphi}$, which means that the spin-dependent splitting occurs also in this direction. Note that the 
spin-dependent splitting will vanish if the beam polarization and inhomogeneous waveplate have the same spatial rotation rate (i.e., $q=m$ ). As the polarization rotation rate of the CVB can be tailored arbitrarily via suitably designing the polarization distribution of the $\mathrm{CVB}$, it can serve as an independent and convenient degree of freedom for manipulating the PBP and photonic SHE.

Experimental results. To demonstrate the role of the inhomogeneous polarization of incident beam in the tunable photonic SHE, a CVB with its polarization exhibiting tunable spatial rotation rate is generated experimentally and passes through a homogeneous waveplate [Fig. 3(a)]. It is a typical example of the Fig. 1(b). Part 1 of the experiment setup can produce a CVB on the equator of the higher-order Poincare sphere where the polarization distribution possesses a cylindrical symmetry ${ }^{25,26}$ [Fig. 3(b)]. The CVB on the equator is generated by interfering the two beams from a modified Mach-Zender interferometer. Two cascaded half-wave plates (HWP2 and HWP3) are employed to determine the polarization distribution of the linearly polarized CVB. By rotating the optical axis directions of the two half-wave plates, we can obtain any desired polarization on the equator of the higher-order Ponicare spheres (See the supplementary materials for the details of the generation of the CVB and calculation of the photonic SHE $)^{19}$. The CVB passes through a homogeneous birefringent waveplate (part 2, HBW) and the Stokes parameter $S_{3}$ is measured by the part 3. Modulating the phase picture displayed on the SLM, we can acquire a CVB with any desired polarization distribution, and thereby introduce a tunable geometric phase gradient even if the light beam propagates through a homogeneous anisotropic waveplate.

The spin-orbit interaction between the CVB and the HBW brings about a tailorable PBP and creates a geometric phase gradient in the azimuthal direction $\hat{e}_{\varphi}$ [see Eqs. (2) and (3)]. This phase gradient can result in a $k$-space spin-dependent splitting also in this direction. And then the real-space splitting manifested as multi-lobe patterns can be directly measured in the far field (Fig. 4), which is attributed to the coherent superposition contributed by the local $k$-space shift in the near field. For $m= \pm 1$, both the splitting patterns have four lobes with 2 -fold rotational $\left(C_{2}\right)$ symmetry. The only difference is their anti-phase distribution of the $S_{3}$ pattern due to their just opposite PBP. The splitting pattern for $m=2$ and 3 respectively show 4 -fold $\left(C_{4}\right)$ and 6-fold $\left(C_{6}\right)$ rotational symmetry. Actually, the lobe number is equal to $4|m|$. As $\varphi$ is the azimuthal angle taking values in the range of 0 to $2 \pi$, $\Phi_{\mathrm{PB}}$ falls in the range of 0 to $4 m \pi$ for $\sigma_{+}$and 0 to $-4 m \pi$ for $\sigma_{-}$, respectively. In the spin-dependent splitting pattern, $\sigma_{+}$and $\sigma_{-}$just has a phase difference of $\pi$, and they respectively repeat again for each $2 \pi$ of $\Phi_{\mathrm{PB}}$. Therefore, the spin-dependent splitting manifests as a pattern of alternative $\sigma_{+}$and $\sigma_{-}$with rotational symmetry, and the total number of the lobes is $4|m|$, which can be switched by modulating the topological charge $(m)$ of the CVB, i.e., changing the phase picture shown on the SLM. Note that if a spin-dependent splitting in the radial direction $\hat{e}_{\mathrm{r}}$ is desired, a CVB with polarization inhomogeneity (and the corresponding PBP gradient) in this direction must be created.

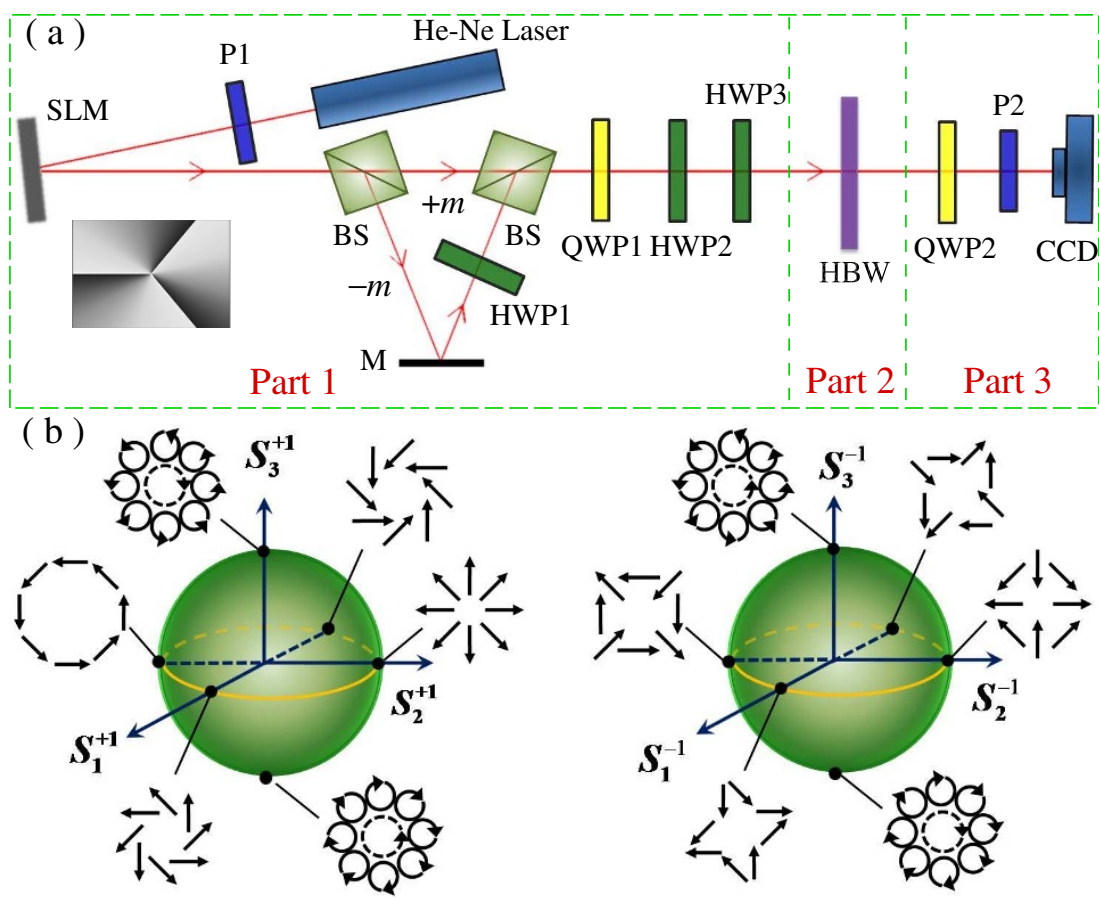

Figure $3 \mid$ (a) Experimental apparatus for generating the CVBs and measuring the tunable photonic SHE. Part 1: A collimated and horizontal polarized $\mathrm{TEM}_{00}$ beam from a He-Ne laser $(632.8 \mathrm{~nm}, 21 \mathrm{~mW})$ followed by a polarizer $(\mathrm{P} 1)$ is converted into a vortex-bearing $\mathrm{LG}_{0, m}$ beam using a reflective phaseonly spatial light modulator (SLM) (Holoeye Pluto-Vis, Germany). Using a modified Mach-Zender interferometer comprised of two beam splitters (BS) and one mirror $(\mathrm{M})$, a $\mathrm{LG}_{0,-m}$ beam is produced by an odd number of reflections. A half-wave plate (HWP1) with $45^{\circ}$ inclined to horizontal direction converts the horizontal polarization to vertical one and a quarter-wave plate (QWP1) gives the two beams orthogonal circular polarizations. Their collinear superposition generates a CVB on the equator of the higher-order Poincaré sphere. In order to determine the concrete point on the equator, two cascaded half-wave plates (HWP2 and HWP3) are employed ${ }^{19,27}$. Part 2: The CVB passes through the homogeneous birefringent waveplate (HBW). The photonic SHE can be detected by Part 3 (a quarter-wave plate followed by a polarizer and a CCD camera) which is a typical setup for measuring the Stokes parameters $S_{3}$. The inset: An example of the phase picture displayed on SLM. (b) Higher-order Poincare spheres. Left panel for the case of $m=1$ and the right panel for $m=-1$. The equator represents the linearly polarized CVBs, the poles circularly polarized vortex beams, and intermediate points between the poles and equator elliptically polarized CVBs. The solid circles with arrows in the north/south poles indicate locally circular polarizations, while the dashed ones represent the direction of vortex. 
(a) $m=-1$
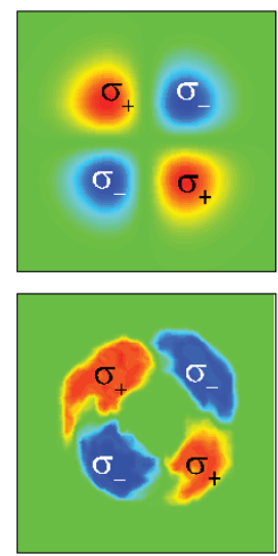

(b) $m=1$
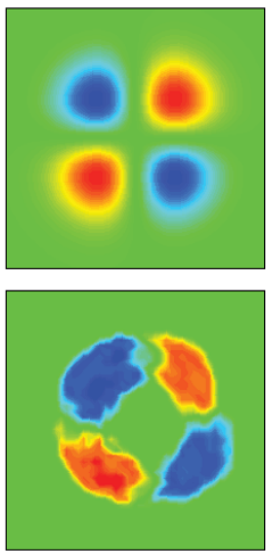

(c) $m=2$
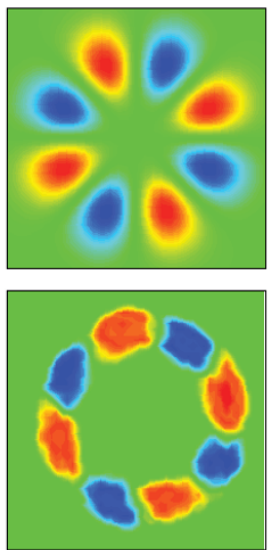

(d) $m=3$
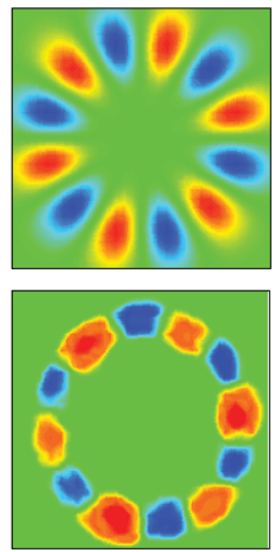

Figure $4 \mid$ Tunable photonic SHE for the CVBs exhibiting different polarization inhomogeneity $m$ under the condition of $q=0$ and $\alpha_{0}=0$. The upper low: theoretical calculations; the lower row: experimental results. Here, $\beta_{0}=0$.

Now let the beam polarization evolve along the equator of the higher-order Poincaré sphere where the polarization has the same spatial rotation rate but different initial polarization angles. When the beams propagating through a homogeneous anisotropic waveplate, the PBP could result in a photonic SHE with rotatable splitting patterns. Figure 5 shows the experimental results of the spin-dependent splitting under the evolution of polarization for $m=1$. Due to the homomorphism between the physical SU(2) space of the light beam and the topological SO(3) space of the higher-order Poincaré sphere, the change of beam polarization of $180^{\circ}$ corresponds to the rotation of $360^{\circ}$ in the equator of the sphere. The splitting lobes also revolve $360^{\circ}$ and return to the original state. This, in turn, indicates the topological characteristic of the photonic SHE.

\section{Discussion}

The inhomogeneous metasurface which can produce a desired transverse phase gradient has been employed to shape the wavefront and manipulate the out-of-plane reflection and refraction of light based on the generalized Snell's law ${ }^{27}$. Therefore, the metasurface can serve as a powerful tool for designing optical components like the inhomogeneous waveplates with locally controllable polarization and geometric phase. The major issues, such as high losses, cost-ineffective fabrications, and unadjustable structures, hamper the development of metasurface technology ${ }^{28}$. But no such difficulty exists when a beam with inhomogeneous polarization is employed to generate the geometric phase. It can produce the same geometric phase gra- dient as the inhomogeneous metasurface does, in particular with a high tunability because a beam with any desired polarization distribution can be conveniently achieved by modulating the phase picture displayed on the SLM (Fig. 3). So, the CVB offers a convenient degree of freedom to manipulate the PBP and photonic SHE.

In summary, tunable photonic SHE has been realized experimentally via tailoring the space-variant PBP produced by a light beam with transversely inhomogeneous polarization. By suitably designing the polarization inhomogeneity, a tunable photonic SHE with any desired spin-dependent splitting (rotatable multi-lobe patterns) is realizable. Though the incident beam with spatial-variant polarization was constructed by a particular method in the experiment, such a scheme has a general meaning in that inhomogeneous polarization distributions provide a robust method to modulate the space-variant PBP, thereby a new way to controllably manipulate the photonic SHE and spin photon. The resulted SHE is large enough for direct measurements, in contrast with the indirect technology using the weak measurement and can be viewed as a photonic version of the famous Stern-Gerlach experiment. Of particular interest is that our results can be generalized to other physical system due to the similar topological origins, such as vortex-bearing electron beam ${ }^{29,30}$.

\section{Methods}

Experimental measurements of the Stokes parameters $S_{3}$. A quarter-wave plate followed by a polarizer and a CCD camera [see the part 3 of the Fig. 3(a)] is a typical setup to measure the Stokes Parameter $S_{3}$ pixel by pixel. By suitably setting the optical axis direction of the quarter-wave plate and the transmission axis of the polarizer, we
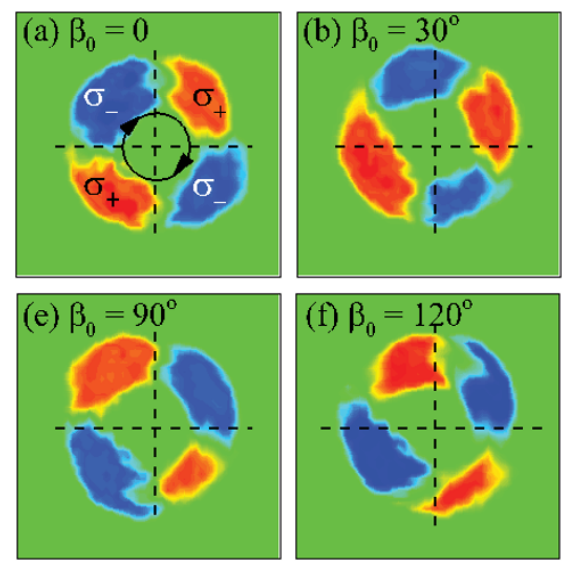
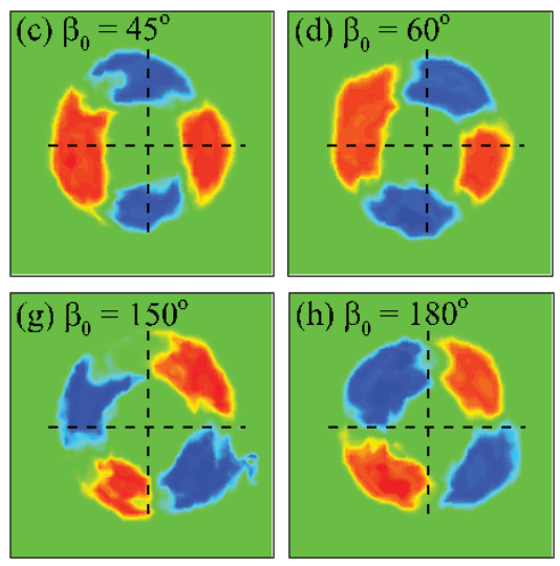

Figure $5 \mid$ Revolving the spin-dependent splitting of the photonic SHE by evolving the polarization of incident CVB along the equator of higher-order Poincré sphere $(m=1)$. The arrows in (a) show the rotation direction. 
can obtain the intensities of the left- and right-handed circular polarization components, respectively. And after a series of calculations, we finally can obtain the $S_{3}$ for each pixel.

1. Murakami, S., Nagaosa, N. \& Zhang, S. C. Dissipationless quantum spin current at room temperature. Science 301, 1348-1351 (2003).

2. Sinova, J. et al. Universal intrinsic spin Hall effect. Phys. Rev. Lett. 92, 126603 (2004).

3. Wunderlich, J., Kaestner, B., Sinova, J. \& Jungwirth, T. Experimental observation of the spin-Hall effect in a two-dimensional spin-orbit coupled semiconductor system. Phys. Rev. Lett. 94, 047204 (2005).

4. Wolf, S. A. et al. Spintronics: a spin-based electronics vision for the future. Science 294, 1488-1495 (2001).

5. Awschalom, D. D. \& Flatte, M. E. Challenges for semiconductor spintronics. Nat. Phys. 3, 153-159 (2007)

6. Chappert, C., Fert, A. \& Dau, F. N. V. The emergence of spin electronics in data storage. Nat. Mater. 6, 813-823 (2007)

7. Onoda, M., Murakami, S. \& Nagaosa, N. Hall effect of light. Phys. Rev. Lett. 93, 083901 (2004)

8. Bliokh, K. Y. \& Bliokh, Y. P. Conservation of angular momentum, transverse shift, and spin Hall effect in reflection and refraction of an electromagnetic wave packet. Phys. Rev. Lett. 96, 073903 (2006).

9. Hosten, O. \& Kwiat, P. Observation of the spin Hall effect of light via weak measurements. Science 319, 787-790 (2008).

10. Zhou, X., Xiao, Z., Luo, H. \& Wen, S. Experimental observation of the spin Hall effect of light on a nanometal film via weak measurements. Phys. Rev. A 85, 043809 (2012).

11. Zhou, X., Ling, X., Luo, H. \& Wen, S. Identifying graphene layers via spin Hall effect of light. Appl. Phys. Lett. 101, 251602 (2012).

12. Shitrit, N. et al. Spin-optical metamaterial route to spin-controlled photonics. Science 340, 724-726 (2013).

13. Bliokh, K. Y, Gorodetski, Y, Kleiner, V. \& Hasman, E. Coriolis effect in optics: unified geometric phase and spin-Hall effect. Phys. Rev. Lett. 101, 030404 (2008).

14. Bliokh, K. Y., Niv, A., Kleiner, V. \& Hasman, E. Geometrodynamics of spinning light. Nat. Photon. 2, 748-753 (2008).

15. Luo, H., Zhou, X., Shu, W., Wen, S. \& Fan, D. Enhanced and switchable spin Hall effect of light near the Brewster angle on reflection. Phys. Rev. A 84, 043806 (2011).

16. Shitrit, N., Bretner, I., Gorodetski, Y., Kleiner, V. \& Hasman, E. Optical spin Hall effects in plasmonic chains. Nano Lett. 11, 2038-2042 (2011).

17. Yin, X., Ye, Z., Rho, J., Wang, Y. \& Zhang, X. Photonic spin hall effect at metasurfaces. Science 339, 1405-1407 (2013).

18. Li, G. et al. Spin-enabled plasmonic metasurfaces for manipulating orbital angular momentum of light. Nano Lett. 13, 4148-4151 (2013).

19. Zhan, Q. Cylindrical vector beams: from mathematical concepts to applications. Adv. Opt. Photon. 1, 1-57 (2009)

20. Marrucci, L., Manzo, C. \& Paparo, D. Optical spin-to-orbital angular momentum conversion in inhomogeneous anisotropic media. Phys. Rev. Lett. 96, 163905 (2006).

21. Niv, A., Gorodetski, Y., Kleiner, V. \& Hasman, E. Topological spin-orbit interaction of light in anisotropic inhomogeneous subwavelength structures. Opt. Lett. 33, 2910 (2008).
22. Ling, X., Zhou, X., Luo, H. \& Wen, S. Steering far-field spin-dependent splitting of light by inhomogeneous anisotropic media. Phys. Rev. A 86, 053824 (2012).

23. Garetz, B. A. Angular doppler effect. J. Opt. Soc. Am. 71, 609-611 (1981).

24. Dahan, N., Gorodetski, Y., Frischwasser, K., Kleiner, V. \& Hasman, E. Geometric Doppler effect: spin-split dispersion of thermal radiation. Phys. Rev. Lett. 105, 136402 (2010).

25. Holleczek, A., Aiello, A., Gabriel, C., Marquardt, C. \& Leuchs, G. Classical and quantum properties of cylindrically polarized states of light. Opt. Express 19, 9714-9736 (2011).

26. Milione, G., Sztul, H. I., Nolan, D. A. \& Alfano, R. R. Higher-order Poincaré sphere, Stokes parameters, and the angular momentum of light. Phys. Rev. Lett. 107, 053601 (2011)

27. Yu, N. et al. Light propagation with phase discontinuities: generalized laws of reflection and refraction. Science 334, 333-337 (2011)

28. Kildishev, A. V., Boltasseva, A. \& Shalaev, V. M. Planar photonics with metasurfaces. Science 339, 1232009 (2013)

29. Bliokh, K. Y., Bliokh, Y. P., Savel'ev, S. \& Nori, F. Semiclassical dynamics of electron wave packet states with phase vortices. Phys. Rev. Lett. 99, 190404 (2007).

30. Karimi, E., Marrucci, L., Grillo, V. \& Santamato, E. Spin-to-orbital angular momentum conversion and spin-polarization filtering in electron beams. Phys. Rev. Lett. 108, 044801 (2012).

\section{Acknowledgments}

This research was supported by the National Natural Science Foundation of China (Grants No. 61025024, No. 11274106, and No. 11347120), the Scientific Research Fund of Hunan Provincial Education Department of China (Grant No. 13B003), and the Doctorial Start-up Fund of Hengyang Normal University (Grant No. 13B42).

\section{Author contributions}

H. Luo initiated the study. The experiment was designed and performed by X.L., X.Z. and W.S. The data analysis was performed by X.L., X.Z. and W.S.; H.L. and S.W. supervised all aspects of the project. All authors contributed to the text of the manuscript.

\section{Additional information}

Supplementary information accompanies this paper at http://www.nature.com/ scientificreports

Competing financial interests: The authors declare no competing financial interests. How to cite this article: Ling, X.H., Zhou, X.X., Shu, W.X., Luo, H.L. \& Wen, S.C. Realization of Tunable Photonic Spin Hall Effect by Tailoring the Pancharatnam-Berry Phase. Sci. Rep. 4, 5557; DOI:10.1038/srep05557 (2014)

This work is licensed under a Creative Commons Attribution-NonCommercialNoDerivs 4.0 International License. The images or other third party material in this article are included in the article's Creative Commons license, unless indicated otherwise in the credit line; if the material is not included under the Creative Commons license, users will need to obtain permission from the license holder in order to reproduce the material. To view a copy of this license, visit http:// creativecommons.org/licenses/by-nc-nd/4.0/ 